\title{
Isoxazole Derivatives With Potential Applications in Polymers and Semiconductors.
}

\author{
Vilela, G. D.; Rosa, R. R. da; Merlo, A. A. \\ LaSOMI, Chemistry Institute, Federal University of Rio Grande do Sul, Bento Gonçalves Avenue, 9500, \\ Campus do Vale, 91501970 Porto Alegre, RS, Brazil \\ *guilherme.drechsler@gmail.com:
}

Keywords: liquid crystal, cycloaddition, isoxazole

\section{INTRODUCTION}

Organic semiconductors have been receiving significant attention as a new class of electronic materials for organic light emitting diodes (OLEDs), organic field-effect transistors (OFETs), and photovoltaic cells.

The success this technologies in contrast with conventional inorganic semiconductors depends on the way of the deposition of the semiconducting layers, the conjugated $\pi$-electron system with high electron affinity, packing geometry; good filmforming properties; chemical stability and purity, etc. These issues are of primary importance in the field of organic electronic materials-OEM.

The purpose of this communication is to show our preliminary results of the synthesis of new liquid crystals through the increase of $\pi$-conjugation using the Pd-catalyzed cross-coupling reactions and increase the molecular stability of crystal SmE phase by polymerization or to prepare tripodal molecules.

\section{RESULTS AND DISCUSSION}

The isoxazolines and isoxazoles core were synthesized in high yields using the Nitrile oxide [3+2] 1,3 -dipolar cycloaddition of aryl nitrile oxide with 4-X-styrenes as dipolarophiles followed by $\mathrm{MnO}_{2}$-oxidation reaction. Using theses reactions we are able to prepare a molecular library including some liquid crystals that have showed SmE mesophase ${ }^{1}$. With those molecules in hands, we chose a liquid-crystalline isoxazole and decided to derive it into two different molecular groups:

- To modify the anisotropy of liquid-crystal and extend the $\pi$-conjugated system by Sonogashira and Suzuki coupling, comparing the results between them.

- To frozen the SmE crystal properties in the glass state by polymerization of the reactive group at the end of the alkyl chain. And, by reaction with aromatic ring trisubstituted a columnar organization could be envisioned with potential jacketing properties.

Figure 1 describe the potential perspective of 3,5diarylisoxzoles as intermediate for organic electronic or plastic electronic materials.

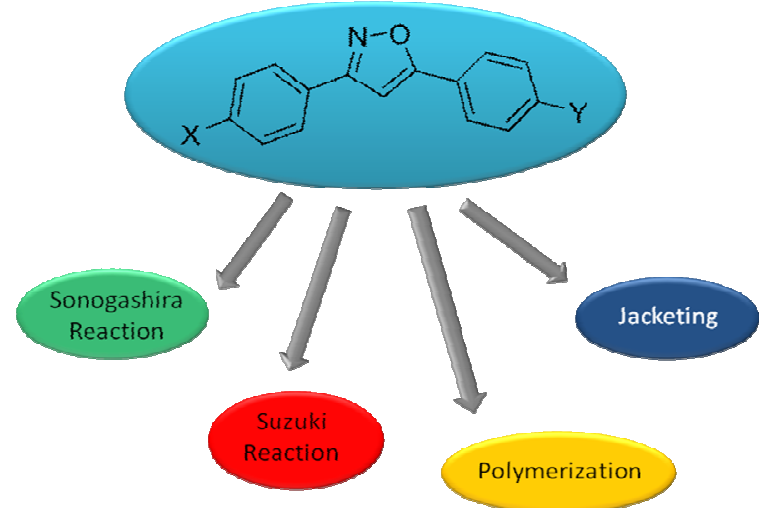

Figure 1. Derivatization of isoxazole into organic and plastic materials.

The data of some intermediates are listed in Table 1. The mesomorphic behavior was analyzed by optical microscopy textures and DSC thermograms.

Table 1. Transitions temperatures for compounds 1-3.

\begin{tabular}{|c|c|c|}
\hline & \\
\hline
\end{tabular}

\section{CONCLUSION}

In conclusion, Pd-catalyzed coupling reactions modified the anisotropy and liquid-crystalline behavior of isoxazole. And we synthesized a "jacketing" precursor to synthesize tripodal molecules.

\section{ACKNOWLEDGEMENTS}

Support: CNPq; CAPES; INCT-CMN; FAPERGS.

\section{REFERENCES}

${ }^{1}$ Vilela, G. D. 2010. M.Sc. PPGQuímica, UFRGS, Porto Alegre, 97.

${ }^{2} \mathrm{He}$-Lou Xie, Chang-Kai Jie, Zhen-Qiang Yu, Xuan-Bo Liu, Hai-Liang

Zhang, J. Am. Chem. Soc., 2010, 132, 8071-8080.

3 Czerniawski, T., Eur. Polymer J., 2000, 36, 635-642. 